\title{
The Logic of the Success/failure System
}

\author{
Dong-Yih Bau \\ Department of Information Management, Da-Yeh University, 168 University Rd., Dacun, \\ Changhua 51591, Taiwan, R.O.C.
}

\begin{abstract}
Understanding the universe is a treasured pursuit of humanity. The success/failure system hypothesis (success/failure system) reflects the mesoscopic structure of the universe, just as quantum mechanics formalizes the microcosmos, and general relativity, the macrocosmos. Herein, we depict the logical analysis of the success/failure system using Einstein's principle theory and apply Russell's analytic philosophy to examine the logical structure of the universe critically. This paper has two goals. First, it aims to show that humanity is able to analyse the cosmos to reveal rationality or intelligence manifested in the universe, as demonstrated by the success/failure system. Second, it emphasizes that the success/failure system may need to be incorporated into our understanding of the cosmos or a final theory of our universe, which would become the triad of quantum mechanics, general relativity and success/failure system.
\end{abstract}

Keywords: Cosmic analysis, Einstein, Final theory, Logical structure of the universe, Russell, Success/failure system

\section{Introduction}

Recently, we used Einstein's principle theory to identify the success/failure system principle and develop the success/failure system hypothesis, ${ }^{1}$ which reflects the mesoscopic structure of the universe, also called the mesocosmos. ${ }^{2}$ Following this work, we applied the success/failure system to our understanding of the cosmos, proposing that the theoretical framework of the cosmos or a final theory of the universe may need to incorporate the success/failure system, in addition to quantum mechanics and general relativity. ${ }^{2}$ A question still remains: how can the scientific community be certain that this principle theory is sufficiently correct and fundamental to feel motivated to partake in this proposal? Our strategy is twofold. First, we look within to conduct a logical analysis of the success/failure system using Einstein's principle theory. ${ }^{3,4}$ Second, we look from outside to examine the logical structure of the universe critically using Russell's analytic philosophy. ${ }^{5-8}$ Our present work is based on its predecessors, ${ }^{1,2}$ which we suggest examining before studying this paper. We also invite the reader to examine Einstein's principle theory ${ }^{3,4}$ and Russell's analytic philosophy ${ }^{5-8}$ to confirm or refute the correctness of our present work.

\section{Logical Analysis using Einstein's Principle Theory}

Einstein's famous aphorism, "The most incomprehensible thing about the universe is that it is comprehensible," $4: 423$ relates to cosmic analysis. We apply his following explanation of how to think in cosmic analysis to the mesocosmos or the success/failure system: ${ }^{1}$ "In speaking here of 'comprehensibility,' the expression is used in its most modest sense. It implies: the production of some sort of order among [the connections of] sense impressions [in their totality], this order being produced [logically] by the [free] creation [of a minimum] of general concepts, relations between these concepts, and by definite relations of some kind between the concepts [and relations] and [the connections of] sense experience[s] [in their totality]."3:292

\subsection{Comprehensibility}

Now, we apply the above to the mesocosmos or the success/failure system. First, we see and experience an order in the universe: at the level of our existence, there can be failures in the universe, which makes it an erring universe. ${ }^{1}$ To reveal the hidden connections of sense impressions in their totality, we freely create minimum general concepts, such as success, failure, part, and whole, ${ }^{1}$ giving sense to 'A part succeeds', 'A part fails', 'The whole succeeds', and 'The whole fails'. Then, we create the relations between these concepts. This brings us to a general fact: if something (the whole) depends on another thing (a part) for its conditions for success, then it depends on that thing for its causes of failure, and vice versa. ${ }^{1}$ We have just created two dependency relations for conditions for success and causes of failure over the part-whole relation.

Second, to account for the complexities of sense experiences and the hidden success/failure connections of sense impressions in their totality, ${ }^{3: 292}$ we require the part-whole relation to be a one-many relation, such that the whole depends on one to many 
parts. We also allow a succession of part-whole relations ad infinitum, which forms a partial ordering structure (PO). ${ }^{1}$ Therefore, the dependency relation of the conditions for success has the properties of reflexivity, antisymmetry, and transitivity, ${ }^{1}$ as does the dependency relation of the causes of failure. ${ }^{1}$ Based on the above analysis, we know rationally the order in the erring universe, as reflected in the logical structure of the universe at this mesocosmic level. We have used the above logical constructions and deductions to achieve a principle theory called the success/failure system principle and hypothesis. ${ }^{1}$ As Einstein said, "In guiding us in the creation of such an order of sense experiences, success alone is the determining factor., $3: 292$

Einstein said, "We do science when we reconstruct in the language of logic [italics for emphasis] what we have seen and experienced" $4: 415$ with "the requirement of logical simplicity.",4:344 We have applied these principles in the logical constructions for the mesocosmos in the preceding two paragraphs. It is remarkable that these logical constructions occur the way they do because any small change in them would lead to logical absurdities. We invite the reader to verify our remark. The reader may, even now, be capable of forming a mental connection between the general fact and the mathematical formulation, Partial Ordering (PO) conditions for success $=$ Partial Ordering (PO) causes of failure, as in Fig. 1 in our first work. ${ }^{1}$ However, in order to understand a principle theory fully and to elucidate the success/failure system's success, we need to understand Einstein's scientific thought ${ }^{3,4}$ concerning the relation between pure reason and experiences, mathematics, and a single theory, as explained below.

\subsection{The Relation between Pure Reason and Experiences}

The eternal antithesis between empiricism and rationalism endures in science. Einstein considered the following: "Pure logical thinking cannot yield us any knowledge of the empirical world; all knowledge of reality starts from experience and ends in it. Propositions arrived at by purely logical means are completely empty as regards reality.... Experience is the alpha and the omega of all our knowledge of reality",3:271 and "[t]here is no logical path to these [universal elementary] laws; only intuition, resting on sympathetic understanding of experience, can reach them."3:226 Thus, the correspondence between our perception of an order of the erring universe and the immediate free creation of the logical elements as to success, failure, part, and whole is without any supporting reason. The same observation was applied to general relativity when Einstein recognized an order of the moving universe and the subsequent free creation of scientific concepts, such as mass, energy, time, and space, even with some clues (e.g., Newton's theory of gravitation) supporting this kind of creation. $^{3,4}$ However, the purely fictitious character of the fundamentals of scientific theory was not recognized by Newton, as Einstein said, "This is no doubt the meaning of [Newton's] saying, hypotheses non fingo. $" 3: 273$

What is the function of pure reason in science? Einstein answered, "We have thus assigned to pure reason and experience their places in a theoretical system of physics [and biology]. The structure of the system is the work of reason; the empirical contents and their mutual relations must find their representation in the conclusion of the theory. In the possibility of such a representation lies the sole value and justification of the whole system, and especially of the concepts and fundamental principles which underlie it."3:272 Thus, the success/failure system hypothesis concludes that every planet or celestial body has the potential to evolve into a success/failure system, and that any empirical system, on Earth and elsewhere, as experienced in privacy by intelligent life, must accord with the success/failure system principle. ${ }^{1}$ This is why Einstein said, "Success [of the creation itself] alone is the determining factor [of a principle theory].

\subsection{Mathematics}

"If, then, it is true that the axiomatic basis of theoretical physics [and biology] cannot be extracted from experience but must be freely invented, can we ever hope to find the right way?"3:274 Einstein answered this question without hesitation "that there is, in my opinion, a right way, and that we are capable of finding it. Our experience hitherto justifies us in believing that nature is the realization of the simplest conceivable mathematical ideas. I am convinced that we can discover by means of purely mathematical constructions the concepts and the laws connecting them with each other, which furnish the key to the understanding of natural phenomena. Experience may suggest the appropriate mathematical concepts, but they most certainly cannot be deduced from it. Experience remains, of course, the sole criterion of the physical [and biological] utility of a mathematical construction. But the creative principle resides in mathematics." $3: 274$

Satisfyingly, our work justifies that the creative principle lies in mathematics. We have used the discrete mathematics of partial ordering (PO) to understand the success/failure system. ${ }^{1}$ The mathematical formulation of the success/failure system principle $^{1}$ is $\mathrm{PO}$ conditions for success $=\mathrm{PO}$ causes of failure. Shall we suggest that partial ordering, ${ }^{1}$ or more precisely, a semi-lattice, is the most beautiful order structure in the universe? Without mathematics, we 
could not justify our confidence that our hypothesis accounts for the mesocosmic view of the universe containing everything or all physical objects. ${ }^{1}$ Each success/failure system has an n-level structure $(\mathrm{n}=2$, $3,4,5 \ldots),{ }^{1}$ and each individual barren celestial body, including stars, planets, asteroids, and satellites, has a one-level structure $(n=1) .{ }^{1}$ From a mathematical point of view, these require only the natural numbers $(\mathrm{n}=1,2,3,4,5 \ldots)$.

\subsection{A Single Theory}

Einstein continued, "In this methodological uncertainty, one might suppose that there were any number of possible systems of theoretical physics [and biology] all equally well justified; and this opinion is no doubt correct, theoretically. But the development of physics [and biology] has shown that at any given moment, out of all conceivable constructions, a single one has always proved itself decidedly superior to all the rest. Nobody who has really gone deeply into the matter will deny that in practice the world of phenomena uniquely determines the theoretical system, in spite of the fact that there is no bridge between phenomena and their theoretical principles; this is what Leibnitz described so happily as a "pre-established harmony.",3:226

In light of the pre-established harmony, it is heartening that the success/failure system is the only known plausible theory that accounts for the mesocosmic view of our universe. If there are two theories or more, we expect that they are logically equivalent to the success/failure system. Indeed, the true difficulty of finding the mesocosmos lies in our inability to imagine it as a principle theory. As Einstein said, "Nature rarely surrenders one of her magnificent secrets!"4:364 and "It is always a blessing when a great and beautiful conception is proven to be in harmony with reality." ${ }^{4: 388}$ We may reflect: how many levels of the universe are there? In each level, may we perceive some logical unity of all phenomena? We appraise the foresight of Einstein's perception of the two great mysteries of the universe, the macrocosmos and the mesocosmos. ${ }^{1}$ We propose that the concepts and relations of success, failure, part, whole, conditions for success and causes of failure may be among the justified and necessary scientific concepts, whether logical or empirical. Furthermore, the success/failure system may need to be incorporated into our understanding of the cosmos or a final theory of our universe, which would become the triad of quantum mechanics, general relativity and the success/failure system. ${ }^{2}$ Over two thousand years ago, the ancient Greeks had already set up an agenda of the cosmos for humanity, which scientists in the twentieth and twenty-first centuries have endeavoured to solve to obtain the ultimate theory. ${ }^{2}$

\section{A Critical Examination using Russell's Analytic Philosophy}

Russell's analytic philosophy can be applied to examine the logical structure of the universe, as revealed by the success/failure system, critically. It can thus facilitate a new understanding of the success/failure system and the mesocosmos. For this purpose, we specifically refer to Russell's analytic philosophy ${ }^{5-8}$ concerning structure, general facts and logical propositions, mathematical logic and Occam's razor. The logical structure of the universe at the mesocosmic level has been solved by Einstein's principle theory ${ }^{3,4}$ and is justified by Russell's analytic philosophy ${ }^{5-8}$ here. The entire gamut of this analytic philosophy is outside the scope of this study, as we only focus on the components relevant to the success/failure system. We will see parallels between Einstein's principle theory ${ }^{3,4}$ and Russell's analytic philosophy ${ }^{5-8}$ and how each can help to explain the other below.

\subsection{Structure}

What exactly does principle theory, which is a logical construction used to comprehend the universe and make inferences, do? According to Russell's analytic philosophy, ${ }^{5-8}$ a principle theory reveals the logical structure of the universe. For example, by seeing a moving universe, general relativity revealed the space-time structure of the universe, whereas by perceiving a jiggling universe, quantum mechanics showed the atomic structure of the universe. By experiencing an erring universe, the success/failure system exposed the success/failure structure of the universe.

For Russell, physics is a self-contained logical system about structure. ${ }^{6: 229}$ Russell's metaphysical world consists of things that have properties, as well as relations to other things. ${ }^{7: 158}$ Structure always involves relations, not properties. 6:223 "The physical world, independently of perception, is known to have a certain structural similarity to the world of our perceptions,"6:138 such as we can imagine. He postulated, "It is because of the importance of structure that theoretical physics is able to content itself with formulae that are about unexperienced occurrences which need not, except in structure, resemble any of the occurrences that we experience., $7: 204$ This justifies principle theory, which consists of attempts to build a bridge between the world of sense and the world of science and to seek structure instead of something vaguely called "causality" or "the uniformity of nature.,"7:205

Thus, the oft-referenced rationality or intelligence manifested in the universe refers to the logical structure of the universe. There are many structures concerning aspects of nature published in 
disciplinary journals. By contrast, the logical structure of the universe is rare at certain levels and unique as a whole. ${ }^{2}$ Whereas general relativity and quantum mechanics depict the quantitative structural contents of the universe, the success/failure system shows its qualitative structural content. ${ }^{2}$ The success/failure system is an important discovery in theoretical science in the twenty-first century and should serve as a fine example of structure when Russell's analytic philosophy is studied.

\subsection{General Facts and Logical Propositions}

Russell's description of the controversy between the two schools called 'empiricists' and 'rationalists' was as follows: "The empiricists - who are best represented by the British philosophers, Locke, Berkeley, and Hume - maintained that all our knowledge is derived from experience; the rationalists - who are represented by the Continental philosophers of the seventeenth century, especially Descartes and Leibniz - maintained that, in addition to what we know by experience, there are certain 'innate ideas' and 'innate principles', which we know independently of experience." $5: 24$

How did Russell's analytic philosophy view the antithesis between empiricism and rationalism? First, Russell considered logic as follows: "In logic, on the contrary, where we are concerned not merely with what does exist, but with whatever might or could exist or be [in the universe], no reference to actual particulars is involved."5:19 Second, Russell distinguished the particular fact from the general fact by saying, "The particularity, in such cases, is that we can imagine general circumstances which would verify our belief, but cannot imagine the particular facts which are instances of the general fact"6:139 and "The reason is that general statements are concerned with intensions, and can be understood without any knowledge of the corresponding extensions." ${ }^{\text {6:139 }}$ For example, "the whole is more than the sum of its parts" is a general fact. Third, Russell still held "that any [logical] proposition other than a tautology, if it is true, is true in virtue to a relation to [general] fact, and that facts in general are independent of experience."7:63,64 Fourth, he distinguished between two kinds of general propositions: empirical generalizations (subject-predicate propositions) and logical propositions (propositions asserting relations). ${ }^{5: 27 ; 7: 171}$ A famous example of empirical generalization is "All men are mortal.",

Now, we are ready to apply Russell's view and treatment to the antithesis between empiricism and rationalism, using the success/failure system as an example. Russell said, "I do not profess to know what the right analysis of general fact is. It is an exceedingly difficult question, and one which I should very much like to see studied." ${ }^{, 771,72}$ In this regard, we believe that Einstein's principle theory and its application to the success/failure system have already studied this question. Russell continued, "This is not a mere [general] fact, but a necessity to which everything actual and possible must conform." ${ }^{, 25}$ For the success/failure system, once the individual components - 'a part succeeds', 'a part fails', 'the whole succeeds', and 'the whole fails' make sense, there necessarily exists the general fact. On the other hand, Aristotle's general fact, "the whole is more than the sum of its parts", seemed to be his free creation. Then, according to Einstein's principle theory, or Russell's analytic philosophy, we may ask Aristotle: what does the universe look like? Russell considered empirical generalizations and logical propositions to be different. The ultimate ground on which "All men are mortal" is based remains inductive, i.e., it is derived from instances. ${ }^{5: 35}$ Aristotle even failed to describe what the universe looks like following his empirical generalization. On the other hand, the logic of the success/failure system ${ }^{1}$ starts by considering what the universe looks like, relates the erring universe to the free creation of concepts and the general fact, and then relates, through deductions, the general fact to the logical proposition, $\mathrm{PO}$ conditions for success $=\mathrm{PO}_{\text {causes }}$ of failure. As Russell said, "Even that part of our knowledge [i.e., logical propositions or principles] which is logically independent of experience (in the sense that experience cannot prove it) is yet elicited and caused by experience"5:24 and "It must be taken as a fact, discovered by reflecting upon our knowledge, that we have the power of sometimes perceiving [and deducing] such relations between universals [i.e., logical elements]...." ${ }^{, 35}$ With logical constructions and deductions based on general facts and logical propositions, the principle theory and the success/failure system have given impartial treatment to empiricism and rationalism.

\subsection{Mathematical Logic}

Initially, Russell created mathematical logic with the aim of adding an empirical basis to pure mathematics as to arithmetic. ${ }^{7: 65}$ In mathematical logic, logical propositions and mathematical propositions are in unity. He later applied the methods of mathematical logic to the interpretation of physics. ${ }^{7: 206} \mathrm{He}$ found that the logic of relations is important in attempting to understand the empirical world. ${ }^{7: 101}$ It seemed to him that those who are not familiar with mathematical logic find great difficulty in understanding the term, 'structure., 7:100

When two relations have the same structure, their logical properties are identical. ${ }^{7: 99}$ For example, in the success/failure system, the two dependency relations, conditions for success and causes of failure, have the three characteristics of reflexivity, antisymmetry and transitivity, and thus have the 
same partial ordering structure. Russell also noted that there must be primitive knowledge of general propositions, which are not acquired by inference, that are used to form premises, and from which, knowledge of general propositions is inferred. ${ }^{8: 70}$ For example, in the success/failure system, ${ }^{1}$ the general fact serves as primitive knowledge and we deduce from it the logical proposition, $\mathrm{PO}$ conditions for success $=$ PO causes of failure. From the mathematical point of view, we anticipate that our understanding of the cosmos or a final theory of our universe may include the three main structures of pure mathematics: topological, algebraic and order.

\subsection{Occam’s Razor}

Although Russell did not say anything regarding a single theory, he said, "Take, for example, the question of waves versus particles [hypothesis for light]....Either hypothesis, therefore, is equally legitimate, and neither can be regarded as having a superior claim to truth. The reason is that the physical world can have the same structure, and the same relation to experience, on the one hypothesis as on the other." ${ }^{, 2225}$ He referred to Einstein's principle theory concerning usage of the principle of Occam's razor ${ }^{7: 268}$ as follows: "The principle may be stated in the form: "Wherever possible, substitute [logical] constructions out of known entities for inferences to unknown entities." "8:130 The referenced principle was "When choosing among different alternative systems of primitive propositions for mathematical logic, 'that one is to be preferred, aesthetically, in which the primitive propositions are fewest and most general'...."7:268 Furthermore, the principle dictated, "Everything said in a science can be said by means of the words in a minimum vocabulary." "6:214 With the quotes constraining the success/failure system, if another theory for the mesocosmos is still discoverable, we expect it to have structural similarity to the success/failure system. While the success/failure system is expected to have a place in our understanding of the cosmos or a final theory of the universe, it may also be superseded by a new and structurally similar hypothesis.

[3] Einstein, A. Ideas and Opinions Bonanza Books: 226, 271-274, 292 (1954).

[4] Calaprice, A. The Ultimate Quotable Einstein Princeton Univ. Press: 344, 364, 388, 415, 423 (2010).

[5] Russell, B. The Problems of Philosophy Project Gutenberg EBook \#5827: 19, 24, 26, 27, 35 (2013).

[6] Russell, B. Human Knowledge: Its Scope and Limits Routledge: 138, 139, 214, 223, 225, 229 (2009).

\section{Conclusions}

Logic is the use of pure thought to understand the world of sense without reference to any particulars. The logical structure of the universe is rarely understood in the literature. Recently, we created the success/failure system to reflect the mesocosmos. In this paper, we use work from two of the most lucid minds of the twentieth century, Einstein's principle theory and Russell's analytic philosophy, to depict the logic of the success/failure system. Based on our logical analysis, we anticipate that the success/failure system may become a permanent part of science. The success/failure system can serve as both a fine example of principle theory by Einstein and an excellent example of analytic philosophy by Russell. We still hold that the success/failure system needs to be incorporated into our understanding of the cosmos or a final theory of our universe, regardless of whether this final theory takes the perspective of 'string theory', 'quantum gravity', 'theory of everything' or some other possibility. To invite the scientific community to understand and conduct research on this hypothesis, we will next sum up our theoretical work, most likely under the title, "The mesocosmos: The success/failure system."

\section{Acknowledgments}

The author would like to thank Anthony Abram for editing and proofreading this manuscript. I am grateful to Suzanna Rona for advice and feedback on the manuscript.

\section{References}

[1] Bau, D. Y. The success/failure system hypothesis. IJASRM 3 (3): 30-34 (2018). http://ijasrm.com/wpcontent/uploads/2018/03/IJASRM_V3S3_496_3 0_34.pdf

[2] Bau, D. Y. The cosmos with the success/failure system. IJASRM 3 (12): 94-97 (2018). http://ijasrm.com/wpcontent/uploads/2018/12/IJASRM_V3S12_1044 _94_97.pdf_http://vixra.org/pdf/1811.0354v1.pdf

[7] Russell, B. My Philosophical Development Spokesman Russell House: 63-65, 99-101, 158, 171, 204-206, 268 (2007).

[8] Russell, B. The Philosophy of Logical Atomism Routledge: 70-72, 130 (2010). 